\title{
Cut and break verbs in Ewe and the causative alternation construction
}

FELIX K. AMEKA and JAMES ESSEGBEY*

\section{Abstract}

Ewe verbs covering the cutting and breaking domain divide into four morpho-syntactic classes that can be ranked according to agentivity. We demonstrate that the highly non-agentive break verbs participate in the causative-inchoative alternation while the highly agentive cut verbs do not, as expected from Guerssel et al.'s (1985) hypothesis. However, four verbs tso 'cut with precision', sế 'cut', lấ 'snap-off', and dze 'split', are used transitively when an instrument is required for the severance to be effected, and intransitively when not. We reject a lexicalist analysis that would postulate polysemy for these verbs and argue for a construction approach.

Keywords: cut and break; separation events; verb semantics; agentivity; causative-inchoative alternation; constructions; Ewe.

\section{Introduction}

Guerssel et al. (1985) group separation verbs into two classes, namely cut verbs and break verbs ( $C \& B$ verbs, hereafter). The two classes are distinguished based on the semantic properties of the verbs, and this in turn supposedly influences their syntactic behavior. For instance, break verbs participate in the causative/inchoative alternation in which the transitive sentence expresses an agent acting on a patient/theme while its intransitive counterpart expresses the theme entering into a state (e.g., John broke the cup vs. the cup broke). Guerssel et al. claim that cut verbs do not participate in the causative/inchoative alternation. Instead, their intransitive counterparts, if a language allows it, are only supposed to be middle constructions (the bread cuts easily). Levin and Rappaport Hovav (1995) attribute the inability of cut verbs to participate in the causative/ 
inchoative alternation to specification of manner and/or instrument in their meaning. In this paper we discuss the $C \& B$ verbs in Ewe in the light of these claims.

Ewe is a cluster of dialects spoken in south-eastern Ghana, Southern Togo, and across the Togo-Benin border. The dialects which are spoken in Ghana are grouped into Southern (e.g., Aylo, Toyu), Central (e.g., Ho, Kpedze), and Northern (e.g., Anfoe, Kpando). The Central and Northern dialects together form the Inland dialects. The data for our paper are drawn from the southern Aylo and the inland Anfoe dialects which are spoken respectively by Essegbey and Ameka, the co-authors.

$\mathrm{C} \& \mathrm{~B}$ verbs in Ewe fall into four classes based on their syntactic behavior. We refer to the classes as highly agentive, agentive, non-agentive, and highly non-agentive. The labels are meant to capture the fact that the semantic property of "agentivity" plays a role in the syntactic behavior of the verbs. The highly agentive and highly non-agentive verbs behave like cut verbs and break verbs respectively, according to the predictions of Guerssel et al. (1985). That is to say while the highly agentive verbs cannot participate in the causative/inchoative alternation, the highly nonagentive verbs can. However, the remaining two classes of verbs participate in the alternation with some restrictions. This raises the issue of how to account for these two classes of verbs. One option would be to posit that each verb has two separate but related meanings, and that each meaning accounts for the occurrence or otherwise of the verb in a construction. In other words one meaning would participate in the alternation while the other would not. An alternative account is to assume that the verbs possess a single core meaning with additional meanings being encoded by constructions, i.e. "form-meaning correspondences that exist independently of the verbs" (cf. Goldberg 1995: 1). We show that data from Ewe favor the second option. The data for our paper were elicited using videoclips showing various acts of separation which were either carried out by an actor or occurred spontaneously (Bohnemeyer et al. 2001, see introduction, Majid et al., this issue, for a description of the stimulus set). Four Aylo and three Anfoe speakers were consulted.

\section{Highly agentive}

The verbs in this class lexicalize instrument and manner or purpose. They are $d z a ́$ 'slash', si 'cut' and kpa 'carve'. Dzá is defined by Westerman (1973: 18) as "to cut with a sharp instrument". We gloss it as 'slash' because it describes cutting events that are done in a slashing manner. The prototypical instruments used to carry out a $d z a$-event are axes or machetes which, because of their heavy nature, have to be swung in the 
process of cutting. While 'slash' suffices to characterize the verb across the dialects, the use differs in Aylo and Anfoe: in Aylo, dzá is only used when there is a slashing event without severance. By contrast, $d z a$ in Anfoe can also describe a slashing event that leads to separation. Thus, Anfoe consultants used $d z a ́$ to describe cutting off a branch with a machete (clip 3) but Aylo consultants rejected it. One Aylo speaker explained:

$\begin{array}{lllll}N \dot{u} \quad y i & k e ́ & w o ́-d z a ́ & m e ́-h i ̃ ́ a & b e ́ \\ \text { thing REL } & \text { INT } & \text { 3PL-slash } & \text { NEG-need that } \\ \text { wò-a-do } & \text { le } & \dot{e}-m e & o .{ }^{1} & \\ \text { 3SG-SUBJ-exit at } & \text { 3SG-inside } & \text { NEG } \\ \text { 'The thing that is slashed need not come apart.' }\end{array}$

Similarly when someone used an axe to chop a piece off a branch (clip 48), the Anfoe, but not Anlo, consultants used dzá. An Aplo consultant did use $d z a ́$ to describe slashing carrots with a machete. Since the carrots were chopped up in the process, one would not have expected the use of $d z a$. His explanation was:

(2) Alé yi wò-ḱ force ḱ apply.
thus REL 3SG-take force take apply
'The way he applied force.'

The focus is on the forcefulness which in turn indicates the manner. Dzá is therefore primarily a manner verb.

Our next verb, si 'cut', describes a cut that is made on the human body with sharp objects like razor blades and knives. Si was therefore used to describe someone cutting her finger with a knife (clip 18). If the cut results in the separation of the body part, however, the Aylos use sế while the Anfoes use lấ (see below). Interestingly, si is also used for some contexts that involve separation. However, in such cases, the severance has a specific purpose. For instance, si be, literally 'cut grass', is used to describe the process of cutting grass to make thatch. In the same vein si de, literally 'cut palm', refers to harvesting palm fruits.

The third verb $k p a$ 'carve' primarily describes the process of carving objects. It therefore requires a sharp instrument. Kpa was not directly elicited with the clips. However, it came up in connection with clip 27 in which a pair of scissors is used to cut off part of the long hair of a Caucasian lady, and clip 30 in which an orange is peeled by hand. Our Anfoe consultants pointed out that when scissors are used to groom a black person's (woolly) hair, the process is rather described as kpa da literally 'carve hair', i.e. stylize hair. Secondly, a common process in Ghana whereby orange sellers use a knife to 'carve out' the rind of the orange is 
also described as kpa. Kpa also describes the use of a sharp object to remove the skin of such tubers as yams and sweet potatoes.

The above verbs all describe events that require the use of a sharp object. In addition, they also incorporate manner and/or purpose. As such, they are highly agentive and do not participate in the causative/ inchoative alternation. Thus, it is unacceptable to say *atía dzá 'the tree slashed', *Kofi fé asi si 'Kofi's hand cut' and *ayutiá kpa 'the orange peeled'.

\section{Agentive verbs}

Agentive verbs, which are tso and sế, describe separations that are primarily carried out with instruments. For instance, tso describes separation of an object that is, or looks as if it was, done with a sharp instrument. Tso was used to describe clips 24 and 49 in which a rope is cut in two with a pair of scissors and a knife respectively, and clip 20 in which a twig is cut off with a knife. Interestingly, using a karate-chop to sever a carrot and a piece of cloth in clips 32 and 34 respectively were also described with tso. The reason is that the hands are shaped in the form of a knife, and the cuts produced look like they were made with sharp objects.

Tso collocates with some entities to describe specific types of cutting. More often than not, performing these cutting events requires some skill. Examples are tso ava 'circumcise', literally 'cut penis', tso lã 'slaughter animal', literally 'cut animal'. Tso is also used to describe the process of cutting fabrics in order to make garments. Because of this, cutting a piece of cloth with a pair of scissors in clip 12 was described with $t s o$. By contrast, tearing a piece of cloth with the hand in clip 1 was not described thus. Tso also describes felling and pruning a tree with an axe or machete. By contrast, where a tree is dug out, as is done for palm trees for the purpose of tapping wine, tso is not used. Finally, tso describes the process of cutting up vegetables like okra for use in a sauce.

We have noted that cuts in flexible objects like ropes were described with $t s o$. Interestingly consultants used the intransitive construction with tso when asked what happened to the rope. Tso was also used to describe a rope snapping spontaneously in clip 46, suggesting that flexible objects that can be severed in a neat way such that it looks as if the cutting was done with a sharp instrument are described with $t s o$. In such cases the focus is on the clean cut, not the instrument. However, where it is clear that the cut can only have been effected with an instrument the verb cannot occur in the intransitive. Thus, although consultants described a rope that snapped in clip 46 as kaa tso 'the rope cut', they rejected *atiá tso 'the tree cut' for describing the end-state of a tree that is cut. 
The second verb in this class, sế, occurs only in the southern dialects. Sế is a general cut verb that describes all cutting events which involve a sharp object. It neither indicates manner nor even entails separation. As a result, it was used to describe several clips. Where consultants wanted to specify that separation is involved, the particle $d a$ ' 'at a distance' was added. For instance, cutting a branch off with a machete (clip 3) was described as follows:

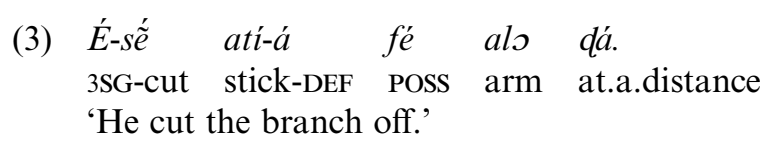

In most cases, however, people contented themselves with using sế alone. There was some hesitation with regard to cutting events that did not involve really sharp instruments. For instance, some speakers used sế to describe clips 32 and 34 in which a carrot and a piece of cloth are separated with a karate-chop respectively. However, it was rejected for clips 23 and 50 in which a hammer is used to sever a piece of cloth and a rope respectively. One could say that while the shape of the karate hand looks like a sharp instrument, the hammer does not. The inland dialects do not have a general cut word that incorporates a sharp instrument. For most of the clips referred to above, the Anfoe consultants used lấ where there was separation. This verb is discussed below.

Sế occurs in the intransitive construction in restricted contexts: clips 13, 24,46 , and 49, which involved the separation of ropes either spontaneously or by an agent, were all described in the intransitive. Speakers used sế to describe clean cuts that could occur by themselves even though they saw it carried out by an agent. Yet where the cut always requires an agent, the intransitive was rejected. Thus clips 3, 9, 26, 32, 37 which involve cutting a branch and some carrots elicited $s \tilde{e}$ in the transitive but not the intransitive. The fact that $t s o$ and $s \tilde{e}$ can occur in the intransitive, however restricted the context, goes contrary to the predictions of Guerssel et al. (1985). We return to this issue in section 5.

\section{Non-agentive}

The non-agentive verbs are lấ 'snap off' and $d z e$ 'split'. Unlike agentive verbs, these ones primarily describe a type of separation. As pointed out in the previous section, lấ is the general cut verb in the inland dialects. It is more general than sế because it does not incorporate any instrument and, for the coastal people, it is only used where there is severance. The example below, which is the description provided for clip 15 in which a 
stick is sawed, shows that a take-SVC can be used to indicate that an instrument is used, and what kind:

(4) É-tsó sá tsó lá́ atí-á.
3SG-take saw take cut stick-DEF
'He cut the stick with a saw.'

Owing to its general meaning lá was used to describe separating a rope with a chisel (clip 2) and an axe (clip 13); and using a karate-chop on a carrot (clip 32), and a rope (clip 61). It was also used to describe pulling and, thereby, breaking a piece off a yarn (clip 38).

Being primarily a severance verb (where severance refers to separation without specification of instrument or manner), lá participates in the causative/inchoative alternation for the most part. For instance, it was used both in the transitive and intransitive constructions to describe separating a cloth with a knife in clip 12 . However, the intransitive was rejected for cutting a branch in clip 3. This is because such branches can only be severed with sharp objects which, in turn, presuppose the presence of an agent. Note that where the branch is separated by being bro-

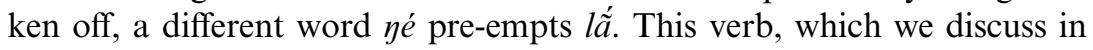
the next section, participates fully in the causative/inchoative alternation.

The second non-agentive verb is $d z e$ 'split'. Dze describes linear separation such as a tear in clothing. It was used to describe the tearing of a piece of cloth in clip 36, and the lengthwise splitting of a carrot with an axe in clip 37. Dze also participates in the causative/inchoative alternation for the most part. Thus, clips 1, 12, 23, and 36 in which a cloth is torn were described with $d z e$ in the transitive and the intransitive. Also, clip 8 in which a piece of cloth is rent without an agent, is described by consultants as awua dze 'the garment split'. However like lấ, some of the separation events described with $d z e$ require an instrument. An example is chopping up firewood, which primarily requires an axe. Although it is acceptable to say $X$ dze náke ' $\mathrm{X}$ split firewood', it is not acceptable to say *nákea dze "the firewood split'. Thus once again, we are confronted with the situation where because a change of state can only have been brought about with an instrument and, therefore, requires an agent, the intransitive construction is ruled out.

The behavior of break verbs like $d z e$ and lấ is not unexpected. Levin and Rappaport Hovav (1995: 102), who consider break verbs to be dyadic, note that they detransitivize when the event they express can "occur spontaneously without volitional intervention of an agent." Thus while the non-agentive verbs, for the most part, describe events that can occur spontaneously and, therefore, participate in the causative/inchoative alternation, there are a few instances in which they describe events that 
require an instrument. In such cases, they do not participate in the alternation.

\section{Highly non-agentive}

The verbs that occur in this class either incorporate the type of object that undergoes a change or the nature of the change. They are vúvu' 'tear', fé 'split', yé 'break' and $g b a$ 'break'. Vúvú, which is vú in the inland dialects, describes tears that occur in flexible objects like paper and cloth. It was used to describe tearing a piece of cloth using the hand in clip 1, and a hammer in clip 23. Vúvú was also used in the intransitive to describe clip 8 in which a piece of cloth divides in two without the action of a causal agent. This is evidence that the verb does not lexicalize instrument.

The best characterization for the next verb, fé 'split' is the linear separation of an object. Fé was used to describe the spontaneous separation in two of a cloth. It is also used to describe the splitting of old trees by themselves in the forest. This is evidence that neither the nature of the object nor the instrument that is used is relevant for fé. The verb was also used to describe the cutting of a melon in two in clip 51. Being round, the melon is not an exemplary object with a lengthwise dimension. Fé is used because the split occurs from the top to the bottom.

The meaning of $f e ́$ is similar to that of $d z e$ which involves separation along a line and which we also gloss as split, and the two verbs overlap heavily in their use. There are some differences though: while all linear separations are $d z e$, only the ones produced along the length of an object are fé. Thus cutting a log crosswise is described as $d z e$ but not fé. Secondly, dze describes minor cuts in flexible objects like cloth while fé describes more substantial cuts. Interestingly, $d z e$ is used to describe splitting an orange with a knife while fé describes the same result but carried out with the fingers.

The final two verbs, $g b a$ and $\eta e$, illustrate an important distinction that Ewe and other African languages make in the description of breaking events, and which is not made in languages like English. Gba describes the breaking of objects like glasses and (earthenware) pots. The focus of the verb is on the cracks that are introduced into the object. Whether the broken pieces come apart or not is of no consequence. Thus a drinking glass that falls and splinters is described in the same way as a pair of reading glasses that falls and cracks. Gba was used to describe the breaking of a pot and a plate in clips 39 and 40 respectively. Consultants were not sure whether it was appropriate for describing the smashing of a carrot and a stick in clips 21 and 31 respectively because these events are not good candidates for the kind of crack that $g b a$ describes. As one consultant 
put it, ati mégbana o 'sticks do not break'. As such, some of them preferred to simply say that the objects have been pounded. However, when pushed as to which of the two break verbs they would accept, they all agreed that $g b a$ was a better rendering of the scenarios than yé.

Dé describes what we refer to as 'fulcrum break' because it involves the snapping of an object along the fulcrum. The break is usually, though not necessarily, caused by some kind of pressure. A classic case is the breaking of a stick across the knee. The types of objects which are susceptible to a $\eta e$-event are sticks and bones. The verb does not describe the breaking of flexible objects like ropes. All four verbs undergo the causative/ inchoative alternation without restriction.

\section{Construction analysis}

Our discussion shows that agentivity plays an important role in determining whether a verb can occur in the intransitive. However, it is difficult to determine the behavior of the verbs based on their lexical specification alone, unless we claim that agentive and non-agentive verbs come in polysemous pairs. In other words, tso 'cut', and sẽ 'cut' would have two different but related meanings, one involving agentive cutting and another involving non-agentive cutting. Apart from being ad-hoc, this analysis ignores an important property of Ewe. On the one hand, highly agentive verbs describe events involving agents only and, therefore, do not occur in the intransitive while, in restricted context, agentive verbs express separations that occur spontaneously and, therefore, participate in the alternation. On the other hand, highly non-agentive verbs do not lexicalize agent at all and, therefore, participate fully in the alternation. Yet non-agentive verbs can describe separations that require an instrument, in which case they do not participate in the alternation. It is clear that the same factor accounts for the behavior of the agentive and non-agentive verbs. Yet they do not belong in the same class. This is shown by the fact that although tso 'cut' (agentive) and lấ 'snap off' (non-agentive) collocate with limbs, e.g., tso ta 'cut off head' and lấ $t a$ 'snap off head', only the latter occurs in the intransitive since it does not require an instrument. The question that arises then is how to capture the common factor that influences the behavior of these two classes of verbs while maintaining their distinctness. As we noted in the introduction, a lexicalist account simply notes that there are two different but related meanings of each of the verbs. Such an account proliferates the lexicon without capturing this commonality.

The above account also ignores an important property of Ewe. It has been established that events that contain "cause" are always expressed 
by a verb and a complement in Ewe (cf. Essegbey 1999). The definition of cause includes bringing about a change in the state of an entity or merely being in control of an action. Thus the expression for 'swim' is fú 'move limb' together with the obligatory complement $t s i$ 'water', while that of 'to eat' is $q u$ 'eat' and the obligatory complement nu' 'thing'. Both events are agentive. The behavior of agentive and non-agentive verbs is significant when viewed against this background; they are transitive when they express cause and intransitive when they don't. We do not need to posit polysemy to account for this. Instead, we propose that the transitive and intransitive sentences in which the verbs occur are instantiations of twoplace and one-place constructions in the sense of Goldberg (1995). The former has a causal meaning while the latter lacks it. A verb that occurs in the two-place construction will always express cause while the one that occurs in the one-place construction will not. The meanings of the verbs themselves do not change.

\section{Conclusion}

We have shown that $\mathrm{C} \& \mathrm{~B}$ verbs in Ewe are grouped into four classes, which we have referred to as highly agentive, agentive, non-agentive, and highly non-agentive. The verbs and the classes to which they belong are shown in the table below:

Table 1. $C \& B$ verbs in Ewe

\begin{tabular}{llll}
\hline Highly agentive & Agentive & Non-agentive & Highly non-agentive \\
\hline$d z a ́$ 'slash' & $t s o$ 'cut' & $l \tilde{a}$ 'snap off' & (vú) vú 'tear' \\
$s i$ 'cut' & sé 'cut' (Anlo) & $d z e$ 'split' & fé 'split' \\
$k p a$ 'carve' & & né 'fulcrum break' \\
& & $g b a$ 'break' \\
\hline
\end{tabular}

Highly agentive and highly non-agentive-verbs behave like cut-verbs and break-verbs respectively. Agentive and non-agentive verbs sometimes behave like cut-verbs, and other times like break-verbs. As a result, they appear to pose a problem for Guerssel et al.'s (1985) hypothesis. We have argued that the grammar of Ewe, which requires that events involving causal agents always occur in the transitive, takes care of this phenomenon. Therefore there is no need to postulate polysemous pairs for these verbs. 


\section{Notes}

* Contact: Felix K. Ameka, Department of African Languages and Cultures, Leiden University, Postbus 9515, 2300 RA, Leiden, The Netherlands, or James Essegbey, Department of African and Asian Languages and Literatures, University of Florida, 458 Grinter Hall, Gainseville, FL 32611-5565, USA. Email adresses:〈F.K.Ameka@let .leidenuniv.nl〉 and 〈essegbey@aall.ufl.edu〉.

1. Abbreviations used are: $3-3^{\text {rd }}$ person; DEF-definite; INT-intensifier; NEG-negative; PL - plural; POss-possessor; SG-singular; sUBJ—subjunctive. High tones only are marked using the acute accent while the grave accent is placed on the pronouns which are orthographically represented thus.

\section{References}

Bohnemeyer, Jürgen, Melissa Bowerman, and Penelope Brown

2001 Cut and break clips. In Levinson, Stephen C., and N. J. Enfield (eds.), Field Manual 2001, Language and Cognition Group, Max Planck Institute for Psycholinguistics. Nijmegen: MPI, 90-96.

Essegbey James

1999 Inherent complement verbs revisited: Towards an account of argument structure in Ewe. Ph.D. thesis, Leiden University. Nijmegen: MPI dissertation series.

Guerssel, Mohamed, Kenneth Hale, Mary Laughren, Beth Levin, and Josie White Eagle 1985 A crosslinguistic study of transitivity alternations. In Eilfort, William H., Paul D. Kroeber, and Karen L. Peterson (eds.), Papers from the Parasession on Causatives and Agentivity at the 21st Regional Meeting. Chicago: Chicago Linguistic Society, 48-63.

Goldberg, Adele

1995 A Construction Grammar Approach to Argument Structure. Chicago: University of Chicago Press.

Levin, Beth, and Malka Rappaport Hovav

1995 Unaccusativity: At the Syntax-Lexical Semantics Interface. Cambridge, MA: MIT Press.

Majid, Asifa, Melissa Bowerman, Miriam van Staden, and James S. Boster

this issue The semantic categories of cutting and breaking events: A crosslinguistic perspective. Cognitive Linguistics 18(2), 133-152.

Westermann, Diedrich

1973 Ewefiala: Ewe-English Dictionary. Nendeln: Kraus Thompson. 